\title{
Influence of incorporating geometric anisotropy on the construction of thematic maps of simulated data and chemical attributes of soil
}

\author{
Luciana Pagliosa Carvalho Guedes ${ }^{1 *}$, Miguel Angel Uribe-Opazo ${ }^{1}$, \\ and Paulo Justiniano Ribeiro Junior ${ }^{2}$
}

The study on spatial variability of soil properties performed through geostatistical techniques allow us to identify the spatial distribution of phenomena by means of a spatial model that considers degree of dependence among observed data, depending on distance and also the direction that separate them, if there is geometric anisotropy, in other words, a directional trend in spatial continuity. However, the main difficulty in decision making regarding the use of anisotropic spatial model focuses on its relevance to the parameters that express the geometric anisotropy in a spatial model exercise in relation to the estimation space. This study aims at identifying the degree of influence of geometric anisotropy on the accuracy of spatial estimation using simulated data sets with different sample sizes and soil chemical properties such as: Fe, potential acidity $(\mathrm{H}+\mathrm{Al})$, organic matter and $\mathrm{Mn}$. Comparing the isotropic and anisotropic models, especially for smaller sample sizes (100 and 169) showed an increased sum of squares of differences between predictions anisotropy factor $\left(F_{a}\right)$ equals 2 . Furthermore, from $F_{a}$ equals 2.5 , over $50 \%$ of the simulations showed values of overall accuracy (OA) of less than 0.80 and values for the concordance index Kappa $(\mathrm{K})$ and Tau $(\mathrm{T})$ from 0.67 to 0.80 , indicating differences between thematic maps. Similar conclusions were obtained for chemical properties of the soil, from $F_{a}$ equals 2 , showing that there are relevant differences regarding the inclusion or not of geometric anisotropy.

Key words: Anisotropy factor, geostatistics, spatial variability.

\section{INTRODUCTION}

If a spatial dependence structure is a function only of the distance separating the locations observed, then the process that describes the spatial variability of the property is said isotropic. However, if this structure differs in relation to the direction, then the process is said anisotropic (Guedes et al., 2008).

Several authors differently classify the types of anisotropy. Isaaks and Srivastava (1989), considering transitive spatial models, i.e. models which have sill (or effective sill) define that when semivariograms show variation in range, sill or both, according to the vary directions, then it is identified the geometric, zonal and combined anisotropy. Journel and Huijbregts (1978) termed as zonal anisotropy, anisotropy of any kind other than geometric. Zimmerman (1993) classifies the types of anisotropy in four groups, based on which parameter model spatial differences occur as you change direction: anisotropy on the sill, in range, the nugget effect and

${ }^{1}$ Universidade Estadual do Oeste do Paraná UNIOESTE, Centro de Ciências Exatas e Tecnológicas PGEAGRI, Cascavel, Paraná, Brasil. *Corresponding author (luciana_pagliosa@hotmail.com).

${ }^{2}$ Universidade Federal do Paraná UFPR (Statistical Laboratory and Geoinformation - LEG), Curitiba, Paraná, Brasil.

Received: 17 January 2013.

Accepted: 16 August 2013

doi:10.4067/S0718-58392013000400013 slope (for models without sill). Furthermore, this author distinguishes two cases the anisotropy in the range: as geometric, when one direction has greater reach; or not geometric, when there are two or more directions with greater spatial continuity, i.e. greater range.

However, there is a consensus of those authors (Journel and Huijbregts, 1978; Isaaks and Srivastava, 1989; Zimmerman, 1993) that relevant differences existing in the directions regarding the nugget effect and sill are little identified and generally do not indicate the presence of anisotropy, but the presence of correlation between the measurement errors and lack of stationarity. Moreover, they report that the most common form of anisotropy among the phenomena with spatial dependence is the geometric anisotropy.

The identification of the existence and type of anisotropy can be preliminarily made by the construction of directional semivariograms (Guedes et al., 2008). Conventional directions used to build these semivariograms are $0^{\circ}, 45^{\circ}, 90^{\circ}$ and $135^{\circ}$, with a tolerance radius of $22.5^{\circ}$, following the system of azimuth direction measurement, considering the North as being the direction $0^{\circ}$, varying the other directions clockwise. However, anisotropic spatial phenomena can be found in other directions (Isaaks and Srivastava, 1989), there being the need for constructing semivariograms in other directions.

Specifically in the case of geometric anisotropy, there 
are two parameters defining this type of anisotropy: the direction of greater spatial continuity $\alpha$, expressed in the azimuth system, and geometric anisotropy factor $F_{a}=a_{2} /$ $a_{1}$, being $F_{a}>1$ (Diggle and Ribeiro Junior, 2007), and the $a_{2}$ is the largest range of the model describing the semivariance function in $\alpha$ direction, which leads to the largest radius of spatial dependence; and $a_{l}$ is the range of the model that describes the semivariance function toward lower spatial continuity $\alpha+90^{\circ}$. The finding of the existence of geometric anisotropy and its subsequent incorporation into the spatial model are of paramount importance for the estimation of non-sampled locations by kriging. Because that will influence the shape of the estimation window to be used in the interpolation process, which will assign greater weight to points sampled towards greater spatial continuity. Therefore, the incorporation of this type of spatial anisotropy in the model will allow for greater accuracy in the construction of thematic maps that describe the spatial variability of the phenomenon under study. There are studies in the literature describing in detail the concept of anisotropy and the construction of anisotropic spatial models (Zimmerman, 1993; Chorti and Hristopulos, 2008; Guedes et al., 2008; Boisvert et al., 2009; Facas et al., 2010). However, the main difficulty of the researchers on the use of anisotropic spatial models focuses on defining the importance of estimated parameters that express the geometric anisotropy in a spatial model (greater continuity angle and anisotropy factor), in relation to its influence on the spatial estimation. Thus, a question inherent to the identification process of anisotropy is: For which set of anisotropic parameters is unreasonable to assume the isotropy assumption?

In this context, the aim of this work is to identify what is the influence of geometric anisotropy in the accuracy of spatial estimation of soil chemical properties. Thus, to validate the results to be obtained for the soil attributes, it will be also evaluated the influence of geometric anisotropy in simulated datasets with different sample sizes and for which values of their anisotropy factor there is a relevant difference in determining thematic maps.

\section{MATERIAL AND METHODS}

For the study of Monte Carlo simulation, four regular sampling configurations were considered: $10 \times 10,13 \times$ $13,15 \times 15,17 \times 17$, with respectively $100,169,225$ and 289 sampling points. To these were simulated datasets, which represent embodiments of multivariate stochastic processes, assuming Gaussian stationary variables, with a linear space model expressed by:

$$
Z\left(\mathrm{~s}_{i}\right)=\mu\left(\mathrm{s}_{i}\right)+\varepsilon\left(\mathrm{s}_{i}\right), \quad i=1, \ldots, n
$$

in which $\mu\left(s_{i}\right)$ is the deterministic term of the model, represented by a constant mean; $Z\left(s_{i}\right)$ corresponds to the observed values of the variable under study in the $n$ known locations, denoted by the vector $s_{i}=\left(x_{i} y_{i}\right)^{t}$, with $i=1, \ldots, n ; \varepsilon\left(s_{i}\right)$ represents the stochastic term with mean zero, i.e. $E\left[\varepsilon\left(\mathbf{s}_{i}\right)\right]=0$, and the variation between points in space, separated by the Euclidean distance $\|\mathbf{h}\|$, such $\mathbf{h}=$ $\mathbf{s}_{i}-\mathbf{s}_{j}$, is determined by a covariance function $C[\|\mathbf{h}\|]=$ $\operatorname{cov}\left[\varepsilon\left(\mathbf{s}_{i}\right), \varepsilon\left(\mathbf{s}_{i}\right)\right], i=1, \ldots, n$.

If the variable under study presents isotropy, then the covariance function is associated with the semivariance function by the following relationship:

$$
\gamma(\|\mathbf{h}\|)=C(\|\mathbf{h}\|)-C(\|\mathbf{0}\|)
$$

In this study the simulated datasets represent Gaussian stationary variables with presence of geometric anisotropy. Thus, it was assumed that:

$$
\gamma\left(\left\|\mathbf{h}^{*}\right\|\right)=\gamma(\|\mathbf{A} \mathbf{h}\|)
$$

with $\mathbf{h}^{*}=\mathbf{A h}=\mathbf{A}\left(\mathbf{s}_{i}-\mathbf{s}_{j}\right)$ expressing a linear transformation (rotation and contraction) applied to $n$ sampling locations, such that:

$$
\mathbf{A}=\left(\begin{array}{cc}
\cos (\alpha) & \sin (\alpha) \\
-\sin (\alpha) / F_{a} & \cos (\alpha) / F_{a}
\end{array}\right)
$$

Thus, for each of the sample configurations were simulated eight datasets from spatial models with geometric anisotropy, considering eight values for the anisotropy factor from an equidistant sequence of values, with lower value equal to 1.5 and greater value equal to 5 . For each combination of sample configuration size an anisotropy factor value were simulated 200 datasets, 100 of those with anisotropic exponential model and 100 with anisotropic spherical model, with semivariance function described in Equation (3), considering the greater spatial continuity angle $(\alpha)$ equals $0^{\circ}$ in the azimuth system. Moreover, were considered in these anisotropic models, the range, nugget effect $\left(\varphi_{1} \geq 0\right)$ and sill $\varphi_{1}+\varphi_{2}$, being $\varphi_{2} \geq$ 0 , denoted respectively by the ordered tender $\left(\varphi_{1}, \varphi_{1}+\varphi_{2}\right.$ a) equals to $(0,10,45)$.

In each simulated dataset, with geometric anisotropic spatial dependence structure, was performed the spatial interpolation by the kriging technique, in a regular sampling grid consisting of 1600 sample points. To perform this interpolation, we first considered the anisotropic model with real parameters. Then, assuming $\alpha$ as the direction of greater spatial continuity, the parameters that define the anisotropic spatial model were estimated by the method of maximum likelihood: anisotropy factor, range, nugget effect and sill. Thus, for the estimate anisotropic model, the spatial prediction of the same mesh with 1600 points previously described was performed.

Furthermore, it was estimated for each simulated dataset, an isotropic model by the method of maximum likelihood, and by means of this model, was performed a spatial prediction of the same sampling grid composed of 1600 points which have been estimated using the anisotropic models, with real and estimated parameters. To determine the influence of the incorporation of geometric anisotropy in the spatial model exerts in the spatial estimation and, to identify to which sample sizes and values of anisotropy factor in the anisotropic model, 
there is a considerable difference in the spatial estimation; the sets of estimated values in the sample grid of 1600 points (considering the isotropic and anisotropic models) were compared on the following measures: square sum of differences of the estimated values and measures of accuracy: Overall accuracy (OA), Kappa Index (K) and Tau concordance index $(\mathrm{T})$, which aim at comparing the thematic maps generated by the two interpolations with the same rating from the error matrix (De Bastiani et al., 2012). The error matrix was constructed, considering 10 intervals of values or classes. Each element of the error matrix represents the area belonging to the class $i$ from the model map and class $j$ from the reference map.

The geostatistical analysis, the identification of anisotropy and the analysis of the influence exerted by the incorporation of spatial anisotropy in the spatial estimation were also performed on a set of real data from an experimental area located in Eloy Gomes Research Center of Cooperativa Central Agropecuária e Desenvolvimento Ltda (COODETEC) in Cascavel, Paraná. The total experimental area of 1.74 ha, with soil classified as Oxysol. This area was divided into a regular grid with 256 plots with dimensions of $7.20 \times 7.20 \mathrm{~m}$, using as sampling design named as stratified systematic unaligned sampling (Souza et al., 1999). Since these 256 plots, 128 plots received localized management and 128 plots had no localized management, using one. The area was planted with soybeans and data used in this work are related to soil chemical properties showed that geometric anisotropy among all chemical attributes surveyed. These being: $\mathrm{Fe}$, potential acidity $(\mathrm{H}+\mathrm{Al})$, organic matter (OM) and Mn, from samples collected in 1998 and 2002, considering the 128 plots that received no localized management.

The data sets under study were obtained by performing routine chemical analysis, performed in the laboratory for soil analysis COODETEC in representative samples from each plot, obtained by mixing four subsamples from the plot, taken in the layer from 0.0 to $0.20 \mathrm{~m}$ depth, within a radius of $0.25 \mathrm{~m}$. Fe and $\mathrm{Mn}$ were extracted by Mehlich-1 with $10 \mathrm{~cm}^{3}$ soil samples to $100 \mathrm{~cm}^{3}$ solution during $16 \mathrm{~h}$ decanting; $\mathrm{H}+\mathrm{Al}$ was obtained in buffer solution of $\mathrm{pH}$ 7.5 SMP; and OM was determined by the Walkley Black method.
Obtaining the simulated datasets and the geostatistical analyzes were made in the software R (R Development Core Team, 2012) using the module geoR (Ribeiro Jr. and Diggle, 2012).

\section{RESULTS AND DISCUSSION}

\section{Analysis of simulated data}

Table 1 shows the percentage of simulations classified into values intervals for the similarity measures, comparing the spatial estimation performed using anisotropic exponential models with actual and estimated parameters. It is noteworthy that the results for the spherical model are not shown as these produced the same conclusions obtained for the exponential model. The intervals at which the values of global accuracy were classified correspond to the minimum level of accuracy $(\mathrm{OA}=0.85)$ to show the similarity between the estimated values (De Bastiani et al., 2012). For the concordance indices of Kappa and Tau, according to Krippendorff (1980), the intervals in which the values of these measures were classified correspond to low, medium and high similarity among the values estimated by the two anisotropic models.

This table shows that even for high values of anisotropy factor (3.5 and 4.5) there is a high similarity between the maps generated with the values obtained by spatial estimation using anisotropic models with actual and estimated parameters. Comparing these measurements it is observed that most of the simulations presented $\mathrm{OA}$ values higher than or equal to 0.85 and values of concordance indices of Kappa and Tau higher than or equal to 0.80 . The same results are presented in the Boxplot graphs shown in Figure 1 for these similarity measures, grouped according to the anisotropy factor, considering all the simulated values of the anisotropy factor. The dashed lines in these graphs indicate the extreme values of the ranges that classify these measures according to the levels of accuracy previously described.

In each of the graphs shown in Figure 1, the first Boxplot corresponds to the referred measures of similarity for every sample size by comparing the spatial estimates of anisotropic models with actual and estimated parameters considering an anisotropy factor equal to 1, i.e. not considering the presence of anisotropy.

Table 1. Percentage of simulations in which their spatial estimation was classified into intervals of values for accuracy measurements, comparing the spatial estimation using the anisotropic model with actual and estimated parameters, grouped according to anisotropy factor $\left(F_{a}\right)$ and simulated sample size.

\begin{tabular}{|c|c|c|c|c|c|c|c|c|c|}
\hline \multirow[b]{2}{*}{$F_{a}$} & \multirow{2}{*}{$\begin{array}{l}\text { Sample } \\
\text { size (n) }\end{array}$} & \multicolumn{2}{|c|}{ Overall accuracy (OA) } & \multicolumn{3}{|c|}{ Kappa (K) } & \multicolumn{3}{|c|}{ Tau (T) } \\
\hline & & {$[0 ; 0.85]$} & {$[0.85 ; 1]$} & {$[0 ; 0.67]$} & {$[0.67 ; 0.8]$} & {$[0.8 ; 1]$} & {$[0 ; 0.67]$} & {$[0.67 ; 0.8]$} & {$[0.8 ; 1]$} \\
\hline \multirow[t]{4}{*}{3.5} & 100 & 18 & 82 & 2 & 13 & 85 & 1 & 10 & 89 \\
\hline & 169 & 4 & 96 & 1 & 2 & 97 & 0 & 2 & 98 \\
\hline & 225 & 2 & 98 & 0 & 1 & 99 & 0 & 1 & 99 \\
\hline & 289 & 0 & 100 & 0 & 0 & 100 & 0 & 0 & 100 \\
\hline \multirow[t]{4}{*}{4.5} & 100 & 18 & 82 & 2 & 12 & 86 & 2 & 8 & 90 \\
\hline & 169 & 6 & 94 & 1 & 4 & 95 & 1 & 2 & 97 \\
\hline & 225 & 1 & 99 & 0 & 0 & 100 & 0 & 0 & 100 \\
\hline & 289 & 1 & 99 & 0 & 1 & 99 & 0 & 1 & 99 \\
\hline
\end{tabular}



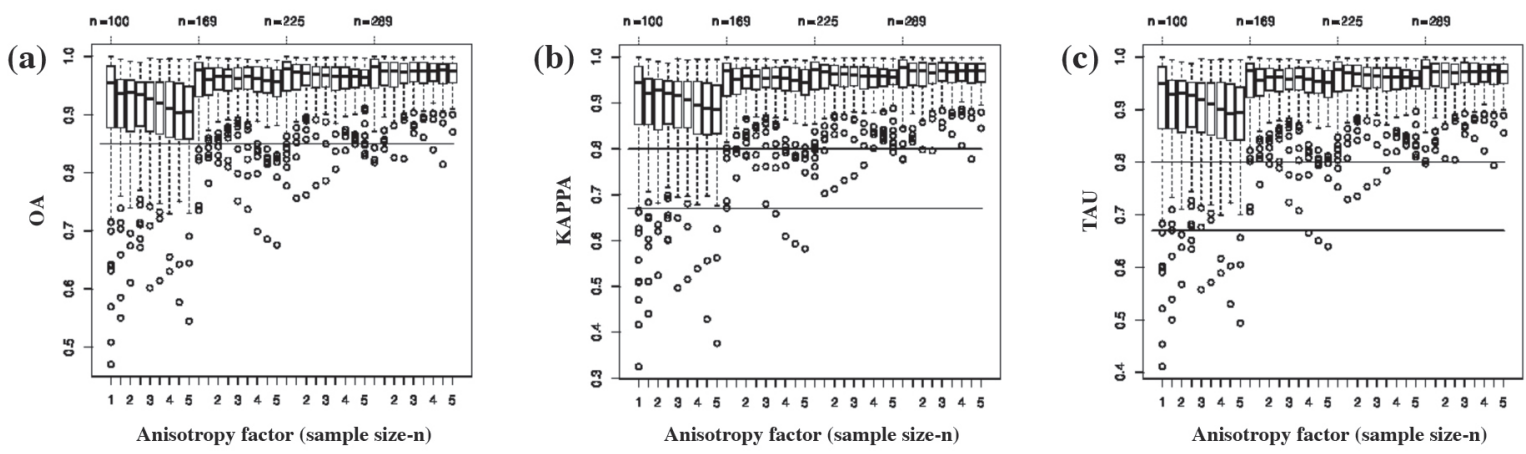

Figure 1. Boxplot graphs grouped by anisotropy factor and sample size for: (a) Overall accuracy (OA), (b) concordance index of Kappa and (c) Tau, relative to comparison of spatial estimations performed by anisotropic spatial model with real and estimated parameters for all simulations.

Thus, when the geometric anisotropy was incorporated into the structure of spatial dependence $F_{a}=1$, it was applied a rotation in spatial coordinates that does not alter the spatial structure under study, considering that the area of spatial dependence is circular and in any direction, there is the same radius of spatial dependence in a model isotropic (Diggle and Ribeiro Jr., 2007). This result is confirmed by the first Boxplot shown in each of the graphs depicted in Figure 1, which shows that more than $75 \%$ of the simulations obtained similarity measures $(\mathrm{OA}, \mathrm{K}$ and $\mathrm{T})$ greater than 0.90 , which indicates high similarity between the maps that would be generated by the spatial estimation.

However, all graphs shown in Figure 1 show that especially for smaller sample sizes (100 and 169) there is a high variability of these measures of similarity, with the presence of some low values (outliers) in approximately $10 \%$ of the simulations. These outliers occurred in simulations where there was an overestimation of the anisotropy factor (ratio between the estimated and simulated factor anisotropy higher than 2).

Tables 2 and 3 show the percentage of simulations classified into values intervals for the similarity measures, comparing the spatial estimation performed using the isotropic exponential model with parameters estimated using anisotropic models with real parameters (Table 2) and with estimated parameters (Table 3), whereas the same intervals of similarity classification described above in Table 1.

Tables 2 and 3 show that for the smallest simulated sample sizes (100 and 169) there was a relevant difference in spatial estimation performed by models that incorporate

Table 2. Percentage of simulations in which their spatial estimation was classified into intervals of values for accuracy measurements, comparing the spatial estimation using the anisotropic model with actual and estimated parameters, grouped according to anisotropy factor $\left(F_{a}\right)$ and simulated sample size.

\begin{tabular}{|c|c|c|c|c|c|c|c|c|c|}
\hline \multirow[b]{2}{*}{$F_{a}$} & \multirow{2}{*}{$\begin{array}{l}\text { Sample } \\
\text { size (n) }\end{array}$} & \multicolumn{2}{|c|}{ Overall accuracy (OA) } & \multicolumn{3}{|c|}{ Kappa (K) } & \multicolumn{3}{|c|}{ Tau (T) } \\
\hline & & {$[0 ; 0.85]$} & {$[0.85 ; 1]$} & {$[0 ; 0.67]$} & {$[0.67 ; 0.8]$} & {$[0.8 ; 1]$} & {$[0 ; 0.67]$} & {$[0.67 ; 0.8]$} & {$[0.8 ; 1]$} \\
\hline \multirow[t]{4}{*}{3.5} & 100 & 100 & 0 & 4 & 88 & 8 & 3 & 84 & 13 \\
\hline & 169 & 77 & 23 & 0 & 50 & 50 & 0 & 42 & 58 \\
\hline & 225 & 69 & 31 & 0 & 50 & 50 & 0 & 25 & 75 \\
\hline & 289 & 61 & 39 & 0 & 15 & 85 & 0 & 96 & 4 \\
\hline \multirow[t]{4}{*}{4.5} & 100 & 100 & 0 & 15 & 1 & 84 & 8 & 4 & 88 \\
\hline & 169 & 93 & 7 & 1 & 78 & 21 & 1 & 70 & 29 \\
\hline & 225 & 91 & 9 & 1 & 72 & 27 & 0 & 52 & 48 \\
\hline & 289 & 73 & 27 & 0 & 57 & 43 & 0 & 75 & 25 \\
\hline
\end{tabular}

Table 3. Percentage of simulations in which their spatial estimation was classified into intervals of values for accuracy measurements, comparing the spatial estimation using the anisotropic model with actual and estimated parameters, grouped according to anisotropy factor $\left(F_{a}\right)$ and simulated sample size.

\begin{tabular}{|c|c|c|c|c|c|c|c|c|c|}
\hline \multirow[b]{2}{*}{$F_{a}$} & \multirow{2}{*}{$\begin{array}{l}\text { Sample } \\
\text { size (n) }\end{array}$} & \multicolumn{2}{|c|}{ Overall accuracy (OA) } & \multicolumn{3}{|c|}{ Kappa (K) } & \multicolumn{3}{|c|}{ Tau (T) } \\
\hline & & {$[0 ; 0.85]$} & {$[0.85 ; 1]$} & {$[0 ; 0.67]$} & {$[0.67 ; 0.8]$} & {$[0.8 ; 1]$} & {$[0 ; 0.67]$} & {$[0.67 ; 0.8]$} & {$[0.8 ; 1]$} \\
\hline \multirow[t]{4}{*}{3.5} & 100 & 86 & 14 & 30 & 51 & 19 & 24 & 48 & 28 \\
\hline & 169 & 79 & 21 & 4 & 56 & 40 & 2 & 44 & 54 \\
\hline & 225 & 68 & 32 & 4 & 42 & 54 & 3 & 30 & 67 \\
\hline & 289 & 53 & 47 & 0 & 32 & 68 & 0 & 19 & 81 \\
\hline \multirow[t]{4}{*}{4.5} & 100 & 91 & 9 & 44 & 44 & 12 & 35 & 50 & 15 \\
\hline & 169 & 84 & 16 & 10 & 67 & 23 & 6 & 64 & 30 \\
\hline & 225 & 84 & 16 & 6 & 61 & 33 & 3 & 53 & 44 \\
\hline & 289 & 70 & 30 & 1 & 53 & 46 & 0 & 43 & 57 \\
\hline
\end{tabular}


or not a geometric anisotropy for the anisotropy factor equal to 3.5 and 4.5. From that anisotropy factor, there was a considerable increase in the percentage of simulations that had overall accuracy values less than 0.85 and index Kappa and Tau with values between 0.67 and 0.8.Thus, through these results and the thematic maps presented in Figures (2 and 3), we have that for smaller sample sizes (100 and 169) and the anisotropy factor equal to 3.5 , there are differences in classifications of generated thematic maps considering isotropic and anisotropic models.

Moreover, the same conclusions are also evidenced in the Boxplot graphs shown in Figures 6 and 7, which describe the values of these measures of accuracy in all simulations, grouped according to anisotropy factor and sample size, comparing the similarity between the maps generated with the isotropic model and anisotropic models.

Thus, these graphs show that for all sample sizes regarded, with increasing anisotropy factor, the values of these measures of accuracy are decreased by comparing the isotropic and anisotropic models. Furthermore, it is evident that for smaller sample sizes (100 e 169), there are differences in the accuracy of measurements from the anisotropy factor of 2.5 , most of the simulations. These results are fundamental for studies on the spatial characteristics of a high cost in sample analysis. Eg, research involving the study of soil properties, whose experiments become impractical for a high sample density (Guedes et al., 2011).

However, Figures (4 and 5) and Figures 6 and 7 show that for larger sample sizes (225 and 289), these accuracy measurements show no significant differences in spatial estimation for anisotropy factors less than 4.5. Figure 8 to 11 show the Boxplot graphs of the sum of squares of the differences between the estimated values of the regionalized variable at non-sampled locations, comparing these models with each other. These graphs show that comparing the spatial estimations made by the anisotropic models (Figure 8a, 9a, 10a and 11a) for the same sample size, the values of the sums of squares were similar, regardless of the value of anisotropy factor, indicating regularity concerning to the spatial difference between the estimations made by anisotropic models with actual and estimated parameters. $90 \%$ of all simulations, regardless of anisotropy factor and sample size, exhibit a sum of squares of differences smaller than 150, including with $F_{a}=1$ that do not alter the spatial structure under study. Furthermore, with increasing sample size, there was reduction of the sum of squares of differences between the estimated values at non-sampled locations using the anisotropic model with actual parameters and the anisotropic model with estimated parameters. Thus, regardless of the value of the anisotropy factor, when there is increased sample size, there is also a greater similarity in spatial estimation, in both models used for this estimation.
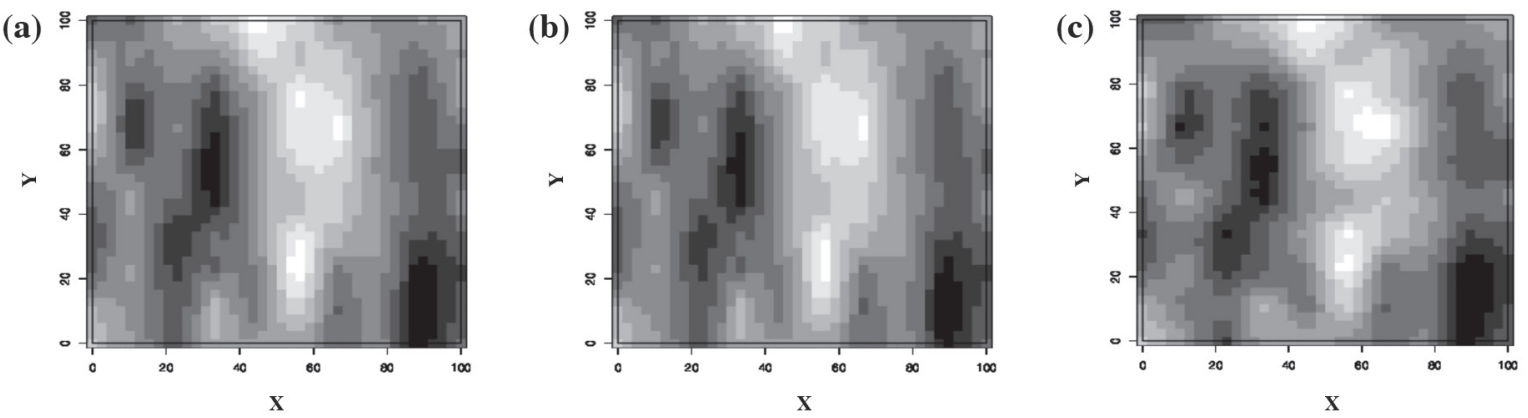

Figure 2. Map of variability for an example of simulated dataset with spatial dependence, $n$ equals 100 and anisotropy factor equal to 3.5, in the spatial estimation model: (a) anisotropic with real parameters; (b) anisotropic with estimated parameters and (c) isotropic with estimated parameters.
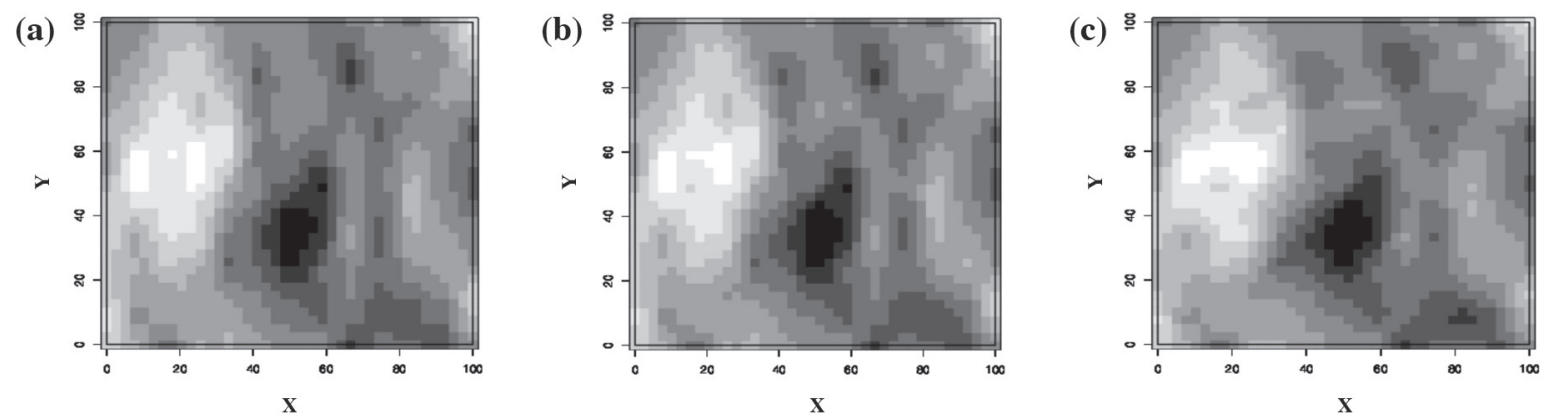

Figure 3. Map of variability for an example of simulated dataset with spatial dependence, $n$ equals 169 and anisotropy factor equal to 3.5, in the spatial estimation model: (a) anisotropic with real parameters; (b) anisotropic with estimated parameters and (c) isotropic with estimated parameters. 
(a)

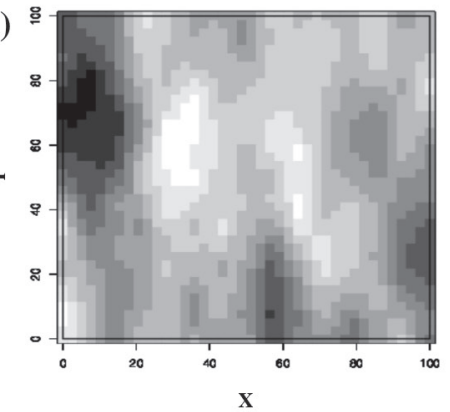

(b)

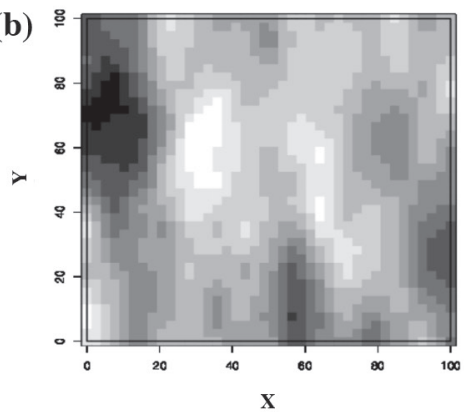

(c)

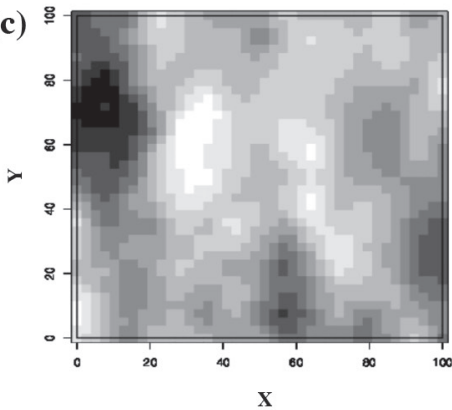

Figure 4. Map of variability for an example of simulated dataset with spatial dependence, $n$ equals 225 and anisotropy factor equal to 3.5 , in the spatial estimation model: (a) anisotropic with real parameters; (b) anisotropic with estimated parameters and (c) isotropic with estimated parameters.
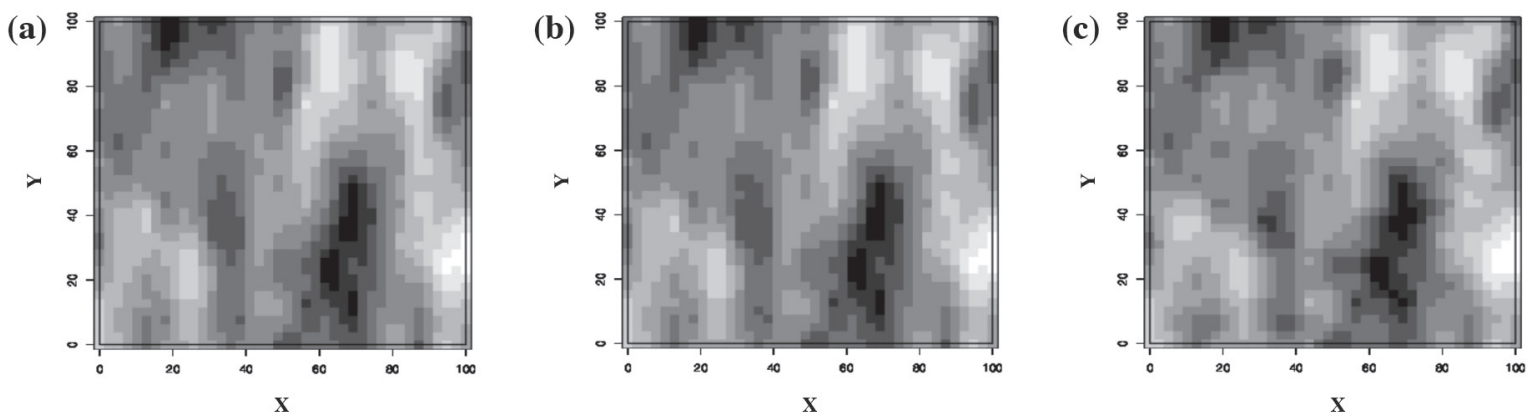

Figure 5. Map of variability for an example of simulated dataset with spatial dependence, $n$ equals 289 and anisotropy factor equal to 3.5 , in the spatial estimation model: (a) anisotropic with real parameters; (b) anisotropic with estimated parameters and (c) isotropic with estimated parameters.

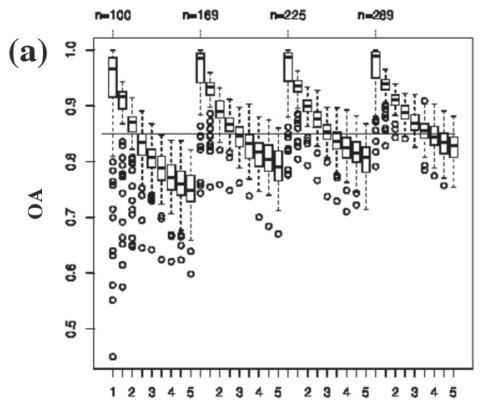

Anisotropy factor (sample size-n)

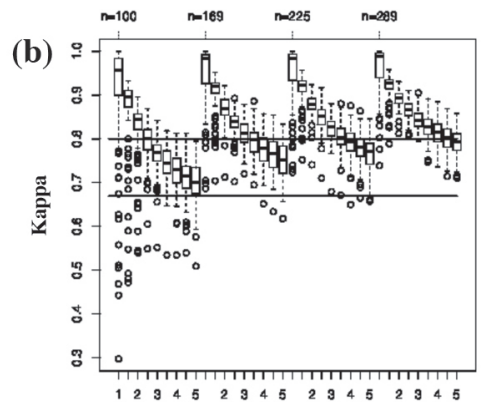

Anisotropy factor (sample size-n)

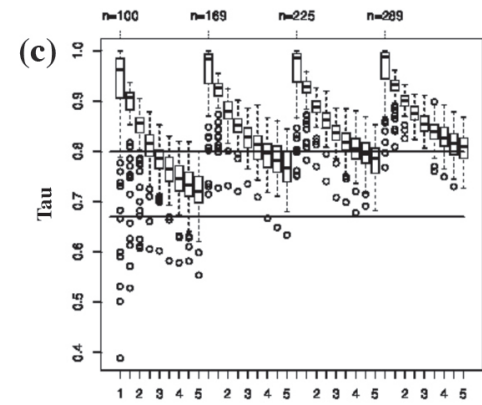

Anisotropy factor (sample size-n)

Figure 6. Boxplot graphs grouped by anisotropy factor and sample size for: (a) Overall accuracy (OA), index of Kappa (b) and Tau (c), comparing the maps generated by the estimated isotropic model and the anisotropic model with real parameters.

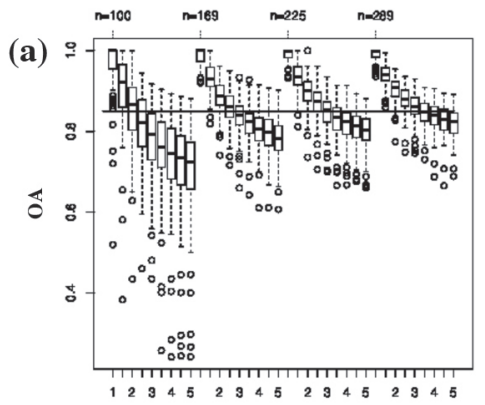

Anisotropy factor (sample size-n)

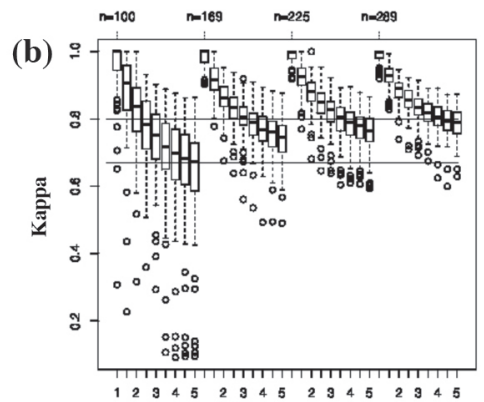

Anisotropy factor (sample size-n)

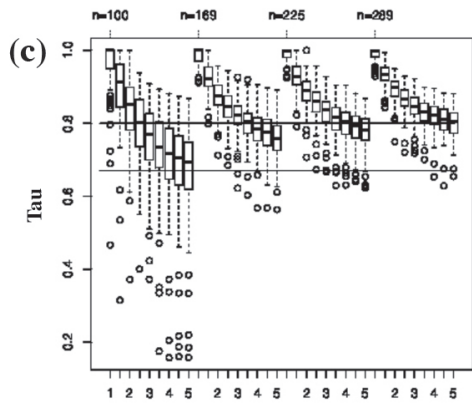

Anisotropy factor (sample size-n)

Figure 7. Boxplot graphs grouped by anisotropy factor and sample size for: (a) Overall accuracy (OA), index of Kappa (b) and Tau (c), comparing the maps generated by the anisotropic and isotropic models with estimated parameters. 
(a)

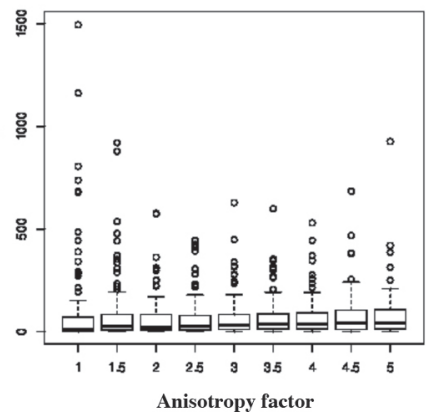

(b)

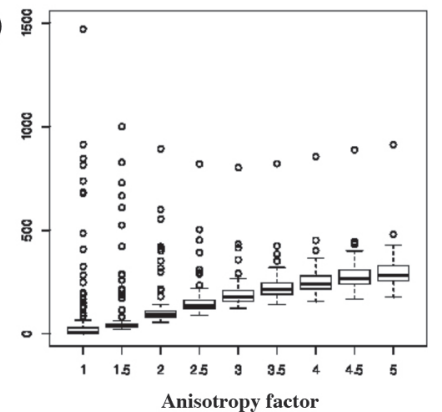

(c)

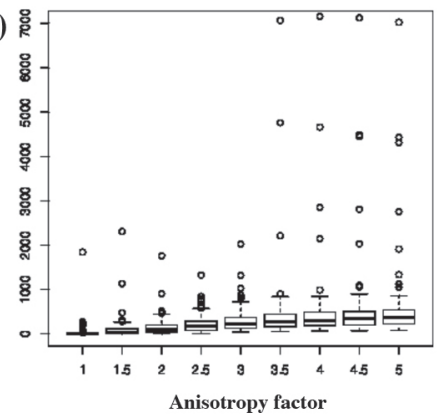

Figure 8. Boxplot graphs grouped by anisotropy factor for sums of squares (SQ) of differences between the spatial estimates performed with 100 sampling points. The figures compare (a) the anisotropic models with actual and estimated parameters by this measure; (b) anisotropic with actual parameters and estimated isotropic and (c) anisotropic and isotropic models with estimated parameters.
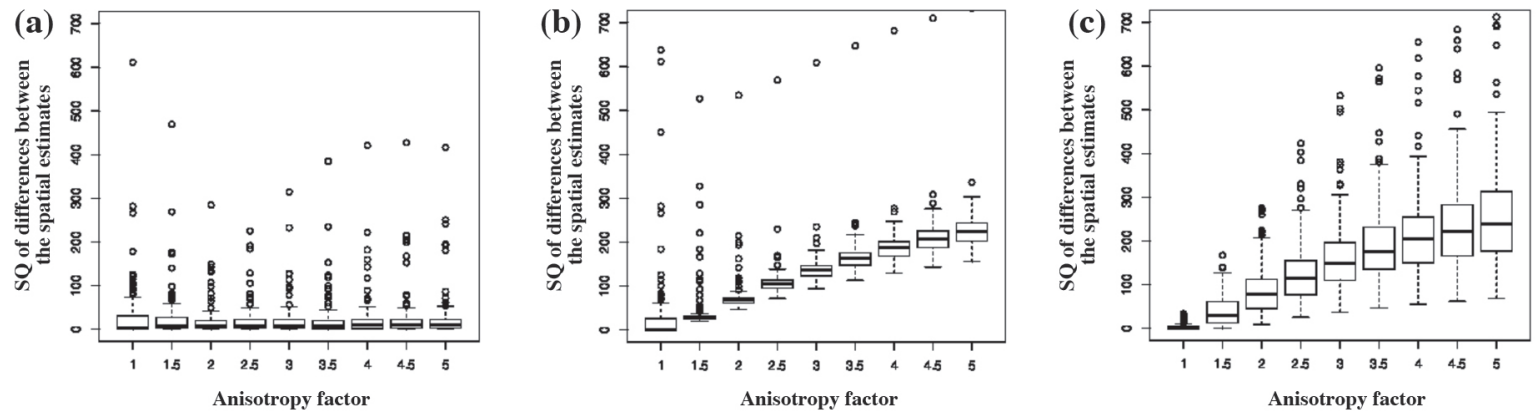

Figure 9. Boxplot graphs grouped by anisotropy factor for sums of squares (SQ) of differences between the spatial estimates performed with 169 sampling points. The figures compare (a) the anisotropic models with actual and estimated parameters by this measure; (b) anisotropic with actual parameters and estimated isotropic and (c) anisotropic and isotropic models with estimated parameters.
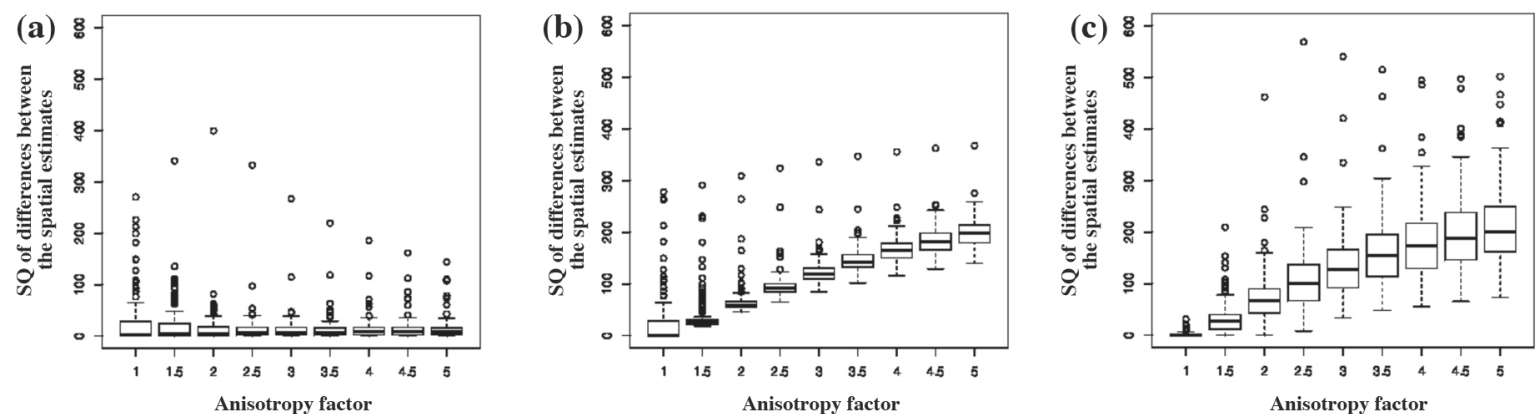

Figure 10. Boxplot graphs grouped by anisotropy factor for sums of squares (SQ) of differences between the spatial estimates performed with 225 sampling points. The figures compare (a) the anisotropic models with actual and estimated parameters by this measure; (b) anisotropic with actual parameters and estimated isotropic and (c) anisotropic and isotropic models with estimated parameters.
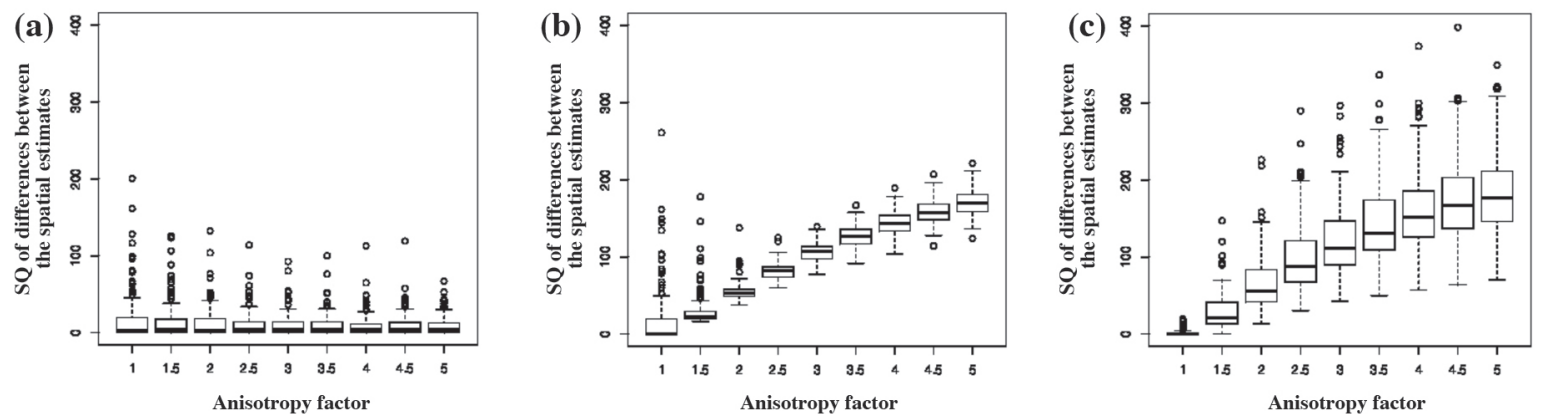

Figure 11. Boxplot graphs grouped by anisotropy factor for sums of squares (SQ) of differences between the spatial estimates performed with 289 sampling points. The figures compare (a) the anisotropic models with actual and estimated parameters by this measure; (b) anisotropic with actual parameters and estimated isotropic and (c) anisotropic and isotropic models with estimated parameters. 
Comparing the sums of squares of the differences between the estimated values at non-sampled locations performed by the anisotropic model with real parameters and isotropic with estimated parameters (Figures 8b, 9b, 10b and 11b), it is observed that regardless of the sample size, with increasing anisotropy factor there was increased sums of squares, mainly from the anisotropy factor equal to 2 .

This increase in the sum of squares is also found by the average rate of variation of the sum of squares of the differences described above, comparing the results obtained for this measure considering the anisotropy factor equal to 1 (no anisotropy) with the other anisotropy factors (Table 4). Thus, it is observed that from the anisotropy factor equal to 2 in all sample sizes, there was a large increase of the average change of the sums of squares, showing significant differences in spatial estimation when considering to their achievement the incorporation or not of geometric anisotropy.

Through the Boxplots of squared sums of differences in spatial estimation, comparing the spatial estimation performed by anisotropic and isotropic models, both with estimated parameters (Figures $8 \mathrm{c}, 9 \mathrm{c}, 10 \mathrm{c}$ and 11c), it is observed that from the sample size equal to 169 and anisotropy factor equal to 1.5 , there was increase of spatial differences between the estimates performed by the two models. These results are also verified by the high values of average rates of variation of differences obtained in

Table 4. Descriptive Statistics of the average rate of change, comparing the results obtained with the anisotropy factor equal to 1 , with the other anisotropy factors compared to the sum of square of differences in spatial estimates, obtained by anisotropic models with real parameters and isotropic with estimated parameters.

\begin{tabular}{|c|c|c|c|c|c|c|c|c|}
\hline \multirow[b]{3}{*}{$F_{a}$} & \multicolumn{8}{|c|}{ Sample size } \\
\hline & \multicolumn{2}{|c|}{100} & \multicolumn{2}{|c|}{169} & \multicolumn{2}{|c|}{225} & \multicolumn{2}{|c|}{289} \\
\hline & Mean & $\mathrm{S}$ & Mean & $\mathrm{S}$ & Mean & $S$ & Mean & $\mathrm{S}$ \\
\hline 1.5 & 5.04 & 197.78 & 20.22 & 90.66 & 22.56 & 51.06 & 21.71 & 42.28 \\
\hline 2.0 & 34.20 & 154.84 & 41.91 & 70.43 & 39.43 & 40.51 & 35.00 & 36.44 \\
\hline 2.5 & 46.96 & 154.84 & 49.56 & 56.63 & 45.85 & 32.60 & 40.79 & 29.06 \\
\hline 3.0 & 52.38 & 106.81 & 52.43 & 45.03 & 47.78 & 27.31 & 42.59 & 23.01 \\
\hline 3.5 & 54.39 & 90.83 & 52.85 & 36.75 & 47.36 & 23.57 & 42.26 & 19.12 \\
\hline 4.0 & 54.58 & 74.92 & 51.86 & 31.03 & 46.14 & 20.44 & 41.03 & 16.31 \\
\hline 4.5 & 53.69 & 69.42 & 50.17 & 27.05 & 44.42 & 18.18 & 39.38 & 14.31 \\
\hline 5.0 & 51.88 & 61.71 & 48.22 & 24.06 & 42.51 & 16.38 & 37.62 & 12.79 \\
\hline
\end{tabular}

S: standard deviation.
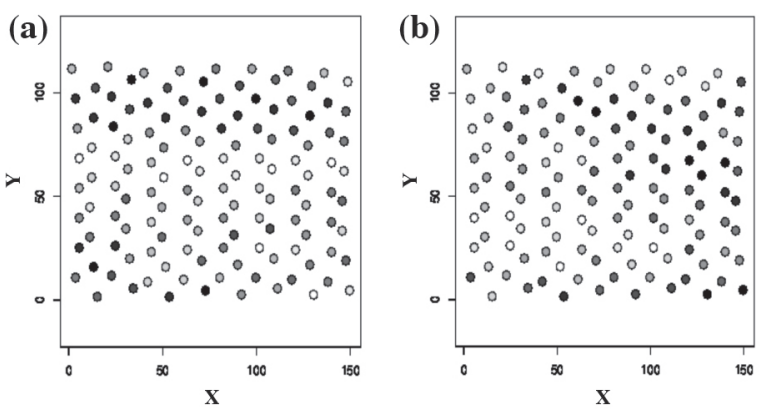

the spatial estimations by comparing the anisotropy factor equal to 1 with the other values for this factor (Table 5). However, in approximately $10 \%$ of the simulations, there were high values of the sums of squares of the differences in spatial estimation, comparing the anisotropic model with estimated parameters and the others. These high values occurred in the simulations that had overestimation of the anisotropy factor.

\section{Analysis of soil chemical properties}

Figure 12 shows the graphs that represent the values of the variables for the sampled plots in the georeferenced study area, and the values of variables are ranked at the same amplitude interval. These graphs shows that the variables $\mathrm{Fe}, \mathrm{OM}$, and $\mathrm{H}+\mathrm{Al}$ (Figures 6a, 6c and 6d) have groups of points with the same classification which extend in the direction of $90^{\circ}$ (azimuth system) indicating the presence of geometric anisotropy in that direction. For variable Mn (Figure 6b), there is a high number of points with the same classification which extend toward $135^{\circ}$ (azimuth system), thus indicating the presence of geometric anisotropy in that direction.

Table 6 shows the results of univariate geostatistical analysis with spatial parameters estimated for the variables $\mathrm{Fe}, \mathrm{Mn}, \mathrm{OM}$, and $\mathrm{H}+\mathrm{Al}$, for isotropic and anisotropic geometric models. According to the criteria of cross-validation and the maximum value of the logarithm

Table 5. Descriptive Statistics of the average rate of change, comparing the results obtained with the anisotropy factor equal to 1 , with the other anisotropy factors compared to the sum of square of differences in spatial estimates, obtained by anisotropic and isotropic models, both with estimated parameters.

\begin{tabular}{|c|c|c|c|c|c|c|c|c|}
\hline \multirow[b]{3}{*}{$F_{a}$} & \multicolumn{8}{|c|}{ Sample size } \\
\hline & \multicolumn{2}{|c|}{100} & \multicolumn{2}{|c|}{169} & \multicolumn{2}{|c|}{225} & \multicolumn{2}{|c|}{289} \\
\hline & Mean & $\mathrm{S}$ & Mean & $\mathrm{S}$ & Mean & $\mathrm{S}$ & Mean & $\mathrm{S}$ \\
\hline 1.5 & 133.60 & 471.04 & 77.13 & 66.01 & 65.78 & 64.79 & 56.11 & 47.01 \\
\hline 2.0 & 128.80 & 213.60 & 86.01 & 55.36 & 73.25 & 55.14 & 65.71 & 36.51 \\
\hline 2.5 & 130.40 & 150.85 & 84.97 & 49.77 & 72.03 & 44.76 & 65.81 & 30.23 \\
\hline 3.0 & 131.70 & 149.27 & 81.25 & 44.80 & 68.61 & 35.08 & 62.05 & 25.06 \\
\hline 3.5 & 156.00 & 327.64 & 76.71 & 39.74 & 64.40 & 28.98 & 57.72 & 21.38 \\
\hline 4.0 & 148.50 & 283.48 & 72.23 & 35.94 & 60.47 & 24.91 & 53.54 & 18.72 \\
\hline 4.5 & 144.00 & 262.29 & 68.00 & 32.89 & 56.76 & 25.91 & 49.83 & 16.65 \\
\hline 5.0 & 131.40 & 225.43 & 64.21 & 30.50 & 53.31 & 19.69 & 46.35 & 14.57 \\
\hline
\end{tabular}

S: standard deviation.

(c)

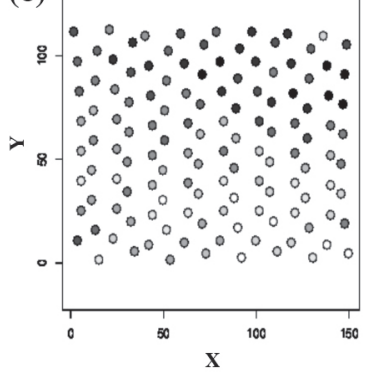

(d)

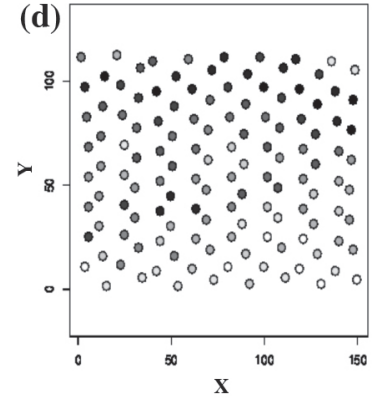

Figure 12. Graph of the spatial representation of plots sampled in the area under study, classified into intervals of equal amplitude for the variables: (a) Fe, (b) Mn, (c) and organic matter (OM) (d) $\mathrm{H}+\mathrm{Al}$. 
Table 6. Spherical spatial models adjusted and the values of their parameters estimated by ML.

\begin{tabular}{llrrrrrr}
\hline Variable & Model & $\hat{\varphi}_{1}$ & $\hat{\varphi}_{1}+\hat{\varphi_{2}}$ & $\hat{a}$ & $\begin{array}{c}\text { EPR } \\
(\%)\end{array}$ & $\alpha$ & $F_{a}$ \\
\hline \multirow{F}{*}{$\mathrm{Fe}$} & Isotropic & 28.19 & 62.10 & 37.85 & 45.39 & & \\
& Anisotropic & 21.89 & 63.15 & 30.55 & 34.66 & $90^{\circ}$ & 9.81 \\
\multirow{2}{*}{$\mathrm{Nn}$} & Isotropic & 15.87 & 97.55 & 36.96 & 16.27 & & \\
& Anisotropic & 12.79 & 109.92 & 33.00 & 11.64 & $135^{\circ}$ & 1.57 \\
$\mathrm{OM}$ & Isotropic & 3.68 & 18.68 & 87.70 & 19.70 & & \\
& Anisotropic & 4.24 & 15.26 & 70.29 & 27.79 & $90^{\circ}$ & 3.66 \\
$\mathrm{H}+\mathrm{Al}$ & Isotropic & 0.33 & 1.49 & 47.50 & 22.14 & & \\
& Anisotropic & 0.17 & 1.50 & 34.42 & 11.33 & $90^{\circ}$ & 3.27 \\
\hline
\end{tabular}

$\hat{a}$ : estimated range; $\hat{\varphi}_{1}$ : estimated nugget effect, $\left(\hat{\varphi}_{1}+\hat{\varphi}_{2}\right)$ : estimated threshold and $\mathrm{EPR}=100 \hat{\varphi}_{1} /\left(\hat{\varphi}_{1}+\hat{\varphi}_{2}\right)$ is the relative nugget effect.

ML: maximum likelihood; OM: Organic matter; EPR: coefficient of relative nugget effect.

of likelihood function, the spherical model was the best fit by the method of maximum likelihood, including the estimates for the anisotropic model, the anisotropy factor. The directions of greater spatial continuity were fixed, considering the conventional directions $0^{\circ}$, $45^{\circ}, 90^{\circ}$ e $145^{\circ}$. In all models, the ranges $(a)$ obtained represent the distance at which the sample points are correlated. According to the values of the nugget effect on the coefficient of relative nugget effect (EPR), that mathematically expresses the proportion of the nugget effect in relation to the sill and indicates the degree of spatial dependence there was strong spatial dependence for $\mathrm{Mn}, \mathrm{H}+\mathrm{Al}$, on all isotropic or anisotropic models and for OM considering the isotropic model (Souza et al. 1999; 0\% EPR $\leq 25 \%$ ). There was a moderate spatial dependence for $\mathrm{Fe}$ in isotropic and anisotropic models and for OM, considering the anisotropic model (Souza et al., $1999 ; 25 \% \leq \mathrm{EPR} \leq 75 \%$ ).
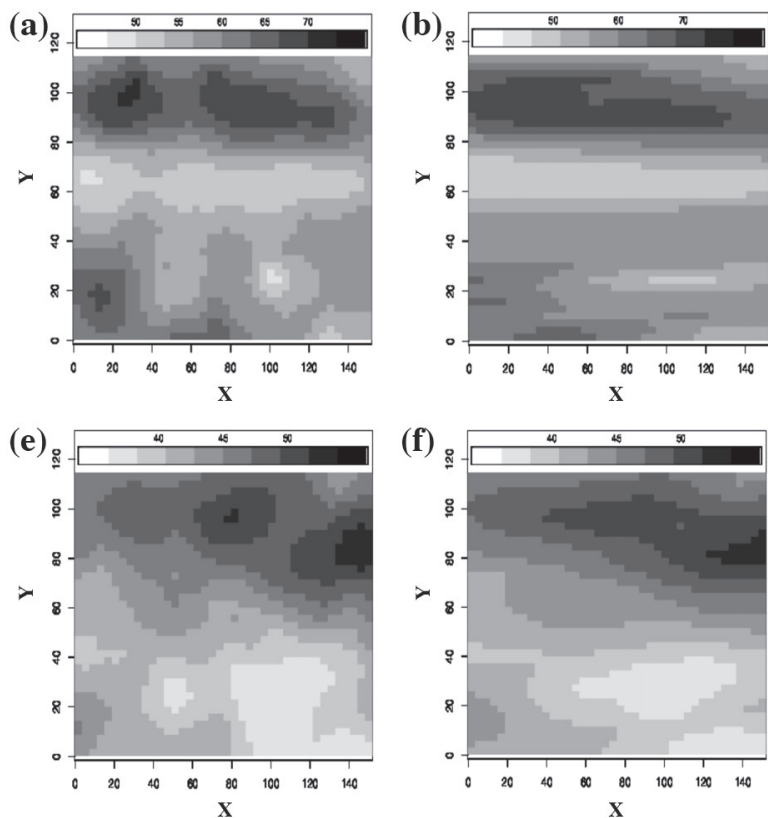

Figure 13. Thematic map of the variables: Fe, with (a) isotropic and (b) geometric anisotropic model; Mn, with (c) isotropic and (d) geometric anisotropic model; organic matter $(\mathrm{OM})$, with (e) isotropic and (f) geometric anisotropic model; and $\mathrm{H}+\mathrm{Al}$, with (g) isotropic and (h) geometric anisotropic model.
Comparing the results of the estimates of parameters of isotropic and anisotropic models, it is observed that only for Mn there was estimated anisotropy factor less than 2 , considering the following directions of greater spatial continuity: $90^{\circ}$ to the variables $\mathrm{Fe}, \mathrm{OM}$ and $\mathrm{H}+$ $\mathrm{Al}$, and $135^{\circ}$ for $\mathrm{Mn}$. Moreover, when incorporated into the geometric anisotropy in the structure expressing the spatial dependence, there is a decrease in the values of estimates of range and a similarity on the sill estimates and nugget effect estimates.

Through results concerning the comparison of estimates obtained by spatial isotropic and anisotropic models (Table 7), there is a small difference between the average variance estimation of spatial, which indicates similarity regarding the reliability in the spatial estimation for the two models (isotropic and anisotropic). However, through the values obtained for similarity measures, it is observed that only the variable $\mathrm{Mn}$, with anisotropy factor estimated less than 2 , showed high similarity between

Table 7. Spherical spatial models adjusted with their respective values of average variance of the spatial estimation $\left(\overline{\sigma^{2}}\right)$ and similarity measures in the comparison between isotropic and anisotropic models to regarding the spatial estimation.

\begin{tabular}{|c|c|c|c|c|c|}
\hline Variable & Model & $\overline{\overline{\sigma^{2}}}$ & $\mathrm{OA}$ & $\mathrm{K}$ & $\mathrm{T}$ \\
\hline \multirow[t]{2}{*}{$\mathrm{Fe}$} & isotropic & 42.54 & 0.78 & 0.74 & 0.76 \\
\hline & anisotropic & 43.65 & & & \\
\hline \multirow[t]{2}{*}{$\mathrm{Mn}$} & isotropic & 43.48 & 0.86 & 0.83 & 0.84 \\
\hline & anisotropic & 36.75 & & & \\
\hline \multirow[t]{2}{*}{ OM } & isotropic & 6.08 & 0.78 & 0.73 & 0.75 \\
\hline & anisotropic & 5.76 & & & \\
\hline \multirow[t]{2}{*}{$\mathrm{H}+\mathrm{Al}$} & isotropic & 0.63 & 0.70 & 0.67 & 0.67 \\
\hline & anisotropic & 0.41 & & & \\
\hline
\end{tabular}

OA: Overall accuracy; concordance index of Kappa (K) and Tau (T); OM organic matter.
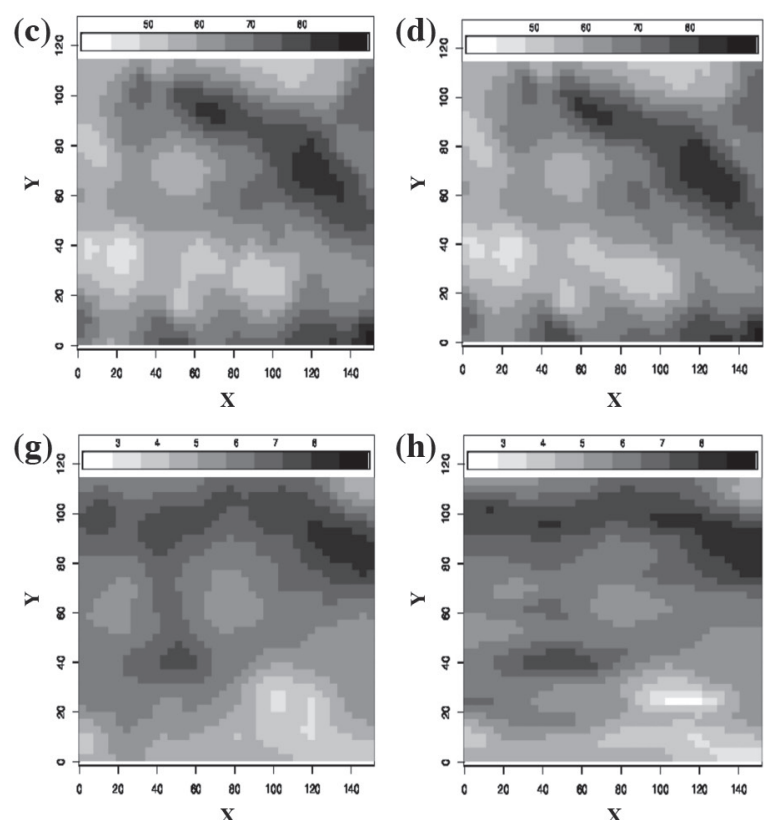
the maps generated by the spatial estimation, with OA value above 0.85 and the concordance indices of $\mathrm{K}$ and $\mathrm{T}$ above 0.80 . The remaining variables that had a estimated anisotropy factor greater than 2, showed values of OA less than 0.85 , indicating a low accuracy regarding the similarity between the maps generated for the estimated values (De Bastiani et al., 2012), and the values of $\mathrm{K}$ and $\mathrm{T}$ between 0.67 and 0.80 ; indicating mean accuracy regarding the maps comparison (Krippendorff, 1980). Thus, it can be verified differences in the classification of thematic maps, regarding the incorporation of geometric anisotropy, and these results are analogous to the results observed in the simulations with smaller sample sizes.

These differences presented in the spatial estimation, regarding the incorporation of geometric anisotropy, can also be visualized on the thematic maps (Figure 13). In thematic maps constructed from the anisotropic models (Figures 13b, 13d, 13f and 13h), there is greater spatial continuity of the subregions in the directions of greater spatial continuity, which are more elliptical. In thematic maps generated by isotropic models (Figures 13a, 13c, $13 \mathrm{e}$, and $13 \mathrm{~g}$ ) in general, the size of the subregions is circular.

\section{CONCLUSIONS}

The analyses performed for the simulations and real data of soil chemical properties showed that when the presence of geometric anisotropy in georeferenced variables is observed, this should be incorporated into the spatial linear model, especially for smaller sample sizes and when the anisotropy factor is greater than two. Therefore, the results obtained in the spatial estimation of these variables in the study area show relevant differences regarding the incorporation or not of anisotropy in the study of spatial variability through the spatial estimation. The incorporation of this feature will produce more reliable estimations that expressed greater detail in the thematic map of the sub-regions, better highlighting the spatial continuity of the attributes under study.

\section{ACKNOWLEDGEMENTS}

CAPES and CNPq for the financial support.

\section{LITERATURE CITED}

Boisvert, J.B., J.G. Manchuk, and C.V. Deutsch. 2009. Kriging in the presence of locally varying anisotropy using non-euclidian distances. Mathematical Geosciences 41:585-601.

Chorti, A., and D.T. Hristopulos. 2008. Nonparametric identification of anisotropic (elliptic) correlations in spatially distributed data sets. IEEE Transactions on signal processing 56:4738-4750.

De Bastiani, F., M.A. Uribe-Opazo, and G.H. Dalposso. 2012. Comparison of maps of spatial variability of soil resistance to penetration constructed with and without covariables using a spatial linear model. Engenharia Agrícola 32:394-404.

Diggle, P.J., and P.J. Ribeiro Jr. 2007. Model-based Geostatistics. 230 p. Springer, New York, USA.

Facas, N.W., M.A. Mooney, M. Asce, and R. Furrer. 2010. Anisotropy in the spatial distribution of roller-measured soil stiffness. International Journal of Geomechanics 10:129-135.

Guedes, L.P.C., M.A. Uribe-Opazo, J.A. Johann, and E.G. Souza. 2008. Anisotropia no estudo da variabilidade espacial de algumas variáveis químicas do solo. Revista Brasileira de Ciência do Solo 32:2217-2226

Guedes, L.P.C., P.J. Ribeiro Junior, S.M.D. Stefano, and M.A. UribeOpazo, 2011. Optimization of spatial sample configurations using hybrid genetic algorithm and simulated annealing. Chilean Journal of Statistics 2: 39-50.

Isaaks, E.H., and R.M. Srivastava. 1989. An introduction to applied geostatistics. 560 p. Oxford University Press, New York, USA.

Journel, A.G., and C.H. Huijbregts. 1978. Mining geostatistics. 600 p. Academic Press, London, UK.

Krippendorff, K. 1980. Content analysis: an introduction to its methodology. 189 p. Sage Publications, Thousand Oaks, California, USA.

R Development Core Team. 2012. R: A language and environment for statistical computing. R Foundation for Statistical Computing Vienna, Austria. ISBN 3-900051-07-0. Available at http:// www.R-project.org (accessed May 2012).

Ribeiro Jr., P.J., and P.J. Diggle. 2012. geoR: A package for geostatistical analysis. R-News 1/2:15-18, 2001 (accessed May 2012).

Souza, E.G., J.A. Johann, J.V. Rocha, S.R.A. Ribeiro, M.S. Silva, and M.A. Uribe-Opazo, et al. 1999. Variabilidade espacial dos atributos do solo em um latossolo roxo distrófico na região de Cascavel-PR. Revista da Sociedade Brasileira de Engenharia Agrícola 8:80-92.

Zimmerman, D. 1993. Another look at anisotropy in geostatistics. Mathematical Geology 25:453-470. 